Www.jmscr.igmpublication.org

Impact Factor (SJIF): 6.379

Index Copernicus Value: 79.54

ISSN (e)-2347-176x ISSN (p) 2455-0450

crossrefDOI: https://dx.doi.org/10.18535/jmscr/v6i10.72

Journal Of Medical Science And Clinical Research

IGM Publication

An Official Publication of IGM Publication

\title{
Menstrual Hygiene and College Absenteeism among Medical Students, Gurugram
}

\author{
Authors \\ Dr Manisha Singh ${ }^{1}$, Dr Manish Kundu, Dr Shalini Ray ${ }^{3 *}$, Dr Abhinav Jain ${ }^{4}$ \\ SGT Medical College, Faculty of Medical And Health Sciences, Gurugram, Haryana \\ *Corresponding Author \\ Dr Shalini Ray \\ Department of Community Medicine, SGT Medical College, Gurugram, Haryana, India \\ Email:drshalini.medhealth@gmail.com
}

\begin{abstract}
Menstrual hygiene has not received substantial attention thereby exposing the females to a gamut of diseases. Though menstruation is a physiological process, it is linked with various false practices and taboos. Dysmennorhoa and premenstrual symptoms are known to restrict the activities of young girls and can also an important cause of absenteeism from work and college. With this backdrop, the present study was conducted among medical students to assess the knowledge among medical students regarding menstrual hygiene and to determine the prevalence of college absenteeism during menstruation and find the prevalence of different pre menstrual symptoms.

Results: The mean age of menarche was $13.38+1.28$ years. Majority of the participants $(82.31 \%)$ practiced good menstrual hygiene and had adequate menstrual hygiene knowledge.

The prevalence of college absenteeism was found to be $45.38 \%$. The main reason for college absenteeism was found to be pain \& discomfort (36.92\%). It was found that most of girls restricted some activities during menstruation the most common being restricted to visit temple (71.54\%). Doctors being the main health care provider should have adequate knowledge about menstrual hygiene and should be an integral part in promotion of reproductive health in the community.

Keywords: Menstrual hygiene, absenteeism, menstruation.
\end{abstract}

\section{Introduction}

According to WHO, menstrual hygiene management (MHM) is the absorption of menstrual blood onto clean material which can be changed in privacy. It also incorporates the availability of soap and clean water, to wash reusable sanitary materials and the body, as well as a suitable place of disposal for used materials (WHO-UNICEF 2012). Menstrual hygiene is not just about the management of the menstrual period but also the need to address societal beliefs and taboos surrounding the issue. Menstruation is associated with various misconceptions, myths and poor practices that can result in adverse health outcomes $^{[1]}$. Menstrual hygiene deals with the special care and requirements of women during their monthly cycle of menstruation ${ }^{[2]}$. MHM is now an integral part of the Swachh Bharat Mission Guidelines (SBM-G) ${ }^{[3]}$. The lack of knowledge and awareness lead to poor personal 
hygiene practices, during menstruation, leading to RTIs.

Absenteeism is a term defined as "the practice of regularly staying away from work or school without good reason"[4]. Dysmenorrhoea is seen to be associated with college absenteeism and restricted participation in various activities. According to studies dysmenorrhea is interrupting the educational and social life of girls. ${ }^{[5]}$ In the United States dysmenorrhea has been estimated to be the greatest cause of time lost from work and school ${ }^{[6]}$. Doctors being main health care provider should have adequate knowledge about menstruation in order to spromote reproductive health.

\section{Objectives}

1. To assess the knowledge, practices regarding menstrual hygiene among female medical students

2. To find prevalence of college absenteeism during menstruation.

3. 3.To find distribution of symptoms of premenstrual syndrome.

\section{Materials and Methods}

The present study was a cross-sectional study which was undertaken in a medical college in Gurugram, Haryana, among the female medical undergraduate students. Study period was from Nov 2016 to Feb 2017.All students except examinee batch present on the day of data collection and giving consent were included in the study. Data was collected using a pre-designed, pre-tested, semi-structured, self-administered anonymous questionnaire with the details of age, age of menarche, knowledge about menstruation, practices and restrictions during menstruation, Pre-menstrual syndrome\& college absenteeism during menstruation. To measure the subject's knowledge on menstrual hygiene, ten questions were used. Each correct response earned 1point, whereas any wrong or don't know response was given no mark; the total score of knowledge was calculated (10 points). Respondents who scored between 0-3points were adjudged as having poor knowledge; whereas those who scored 4-8 were adjudged as having average knowledge and $>8$ as good. ${ }^{[7]}$ Statistical analysis was done using simple proportions,

\section{Results}

The mean age of study participants was $19.95 \pm 1.41$ years. The mean age of menarche was $13.38 \pm 1.28$ years. Majority of the participants $(82.31 \%)$ practiced good menstrual hygiene and had adequate menstrual hygiene knowledge. The participants' knowledge of menstruation and its hygiene management was scored using a scoring system adopted from previous study. ${ }^{[7]}$

Table 2 shows the level of knowledge among study participants regarding menstruation. Majority $82.31 \%$ of the participants had good level of knowledge, followed by $16.15 \%$ who had average knowledge and rest $1.54 \%$ had poor knowledge.

Table 3 shows the different practices during menstruation among study participants. Most of the girls followed good practices during menstruation including appropriate disposal of absorbent, regular changing of sanitary pads, cleaning of private areas etc.

The prevalence of college absenteeism was found to be $45.38 \%$. The main reason for college absenteeism was found to be pain \& discomfort $(36.92 \%)$ followed by unhygienic toilets (21.54\%), lack of water supply in toilets $(13.85 \%)$, fear of staining $(6.15 \%)$, lack of dustbin $(1.54 \%)$ and lack of privacy in toilets $(0.77 \%)$ as shown in figure 1 .

Figure 2 shows the restriction of activities among study participants during menstruation. It was found that most of girls restricted some activities during menstruation the most common being restricted to visit temple $(71.54 \%)$, inability to do sport $(66.15 \%), 43.85 \%$ girls were unable to walk, $35.38 \%$ preferred to stay at home and $33.85 \%$ girls restricted to do daily activities .

Figure 3 shows the distribution of premenstrual symptoms among study participants. Irritability 
was found to be the most common premenstrual symptom (80\%) followed by mood swings (74.62\%), fatigue, abdominal bloating, anxiety etc.

Table 1: Knowledge Regarding Menstruation among study participants

\begin{tabular}{|l|c|}
\hline Questions & Percentage \\
\hline $\begin{array}{l}\text { Heard about menstruation before attaining } \\
\text { menarche }\end{array}$ & $76.15 \%$ \\
\hline Feel comfortable to talk about menstruation & $86.92 \%$ \\
\hline Aware about the ideal age range of menarche & $66.92 \%$ \\
\hline $\begin{array}{l}\text { Aware about the normal menstruation bleeding } \\
\text { duration }\end{array}$ & $80 \%$ \\
\hline Knew normal duration of menstrual cycle & $93.85 \%$ \\
\hline Menstruation is a physiological process & $99.23 \%$ \\
\hline Role of hormones in menstruation & $96.15 \%$ \\
\hline Knew menstruation blood comes from Uterus & $97.7 \%$ \\
\hline $\begin{array}{l}\text { Knew woman can't conceive during } \\
\text { menstruation }\end{array}$ & $92.31 \%$ \\
\hline $\begin{array}{l}\text { Aware sanitary pads are used as a menstrual soak } \\
\text { up }\end{array}$ & $98.46 \%$ \\
\hline
\end{tabular}

Table 2: Knowledge scoring among study participants regarding menstruation

\begin{tabular}{|l|c|c|}
\hline Knowledge & Frequency & Percent \\
\hline average & 42 & $16.15 \%$ \\
\hline good & 214 & $82.31 \%$ \\
\hline poor & 4 & $1.54 \%$ \\
\hline Total & 260 & $100.00 \%$ \\
\hline
\end{tabular}

Table 3: Practices during Menstruation Among Study Participants

\begin{tabular}{|l|c|}
\hline \multicolumn{1}{|c|}{ Practices } & Percentage \\
\hline Wash genitals daily & $97.69 \%$ \\
\hline $\begin{array}{l}\text { Cleaning of genitals with soap or vaginal } \\
\text { wash }\end{array}$ & $83.08 \%$ \\
\hline $\begin{array}{l}\text { Cleaning of undergarments during } \\
\text { menstrual days }\end{array}$ & $97.69 \%$ \\
\hline Cleaning of pubic hair & $92.31 \%$ \\
\hline Use of sanitary pad & $98.46 \%$ \\
\hline Dispose of absorbent in dustbin after changing & $99.23 \%$ \\
\hline $\begin{array}{l}\text { Washing of hands aft } \\
\text { absorbent }\end{array}$ \\
\hline
\end{tabular}

Figure 1: Reasons for College Absenteeism during Menstruation

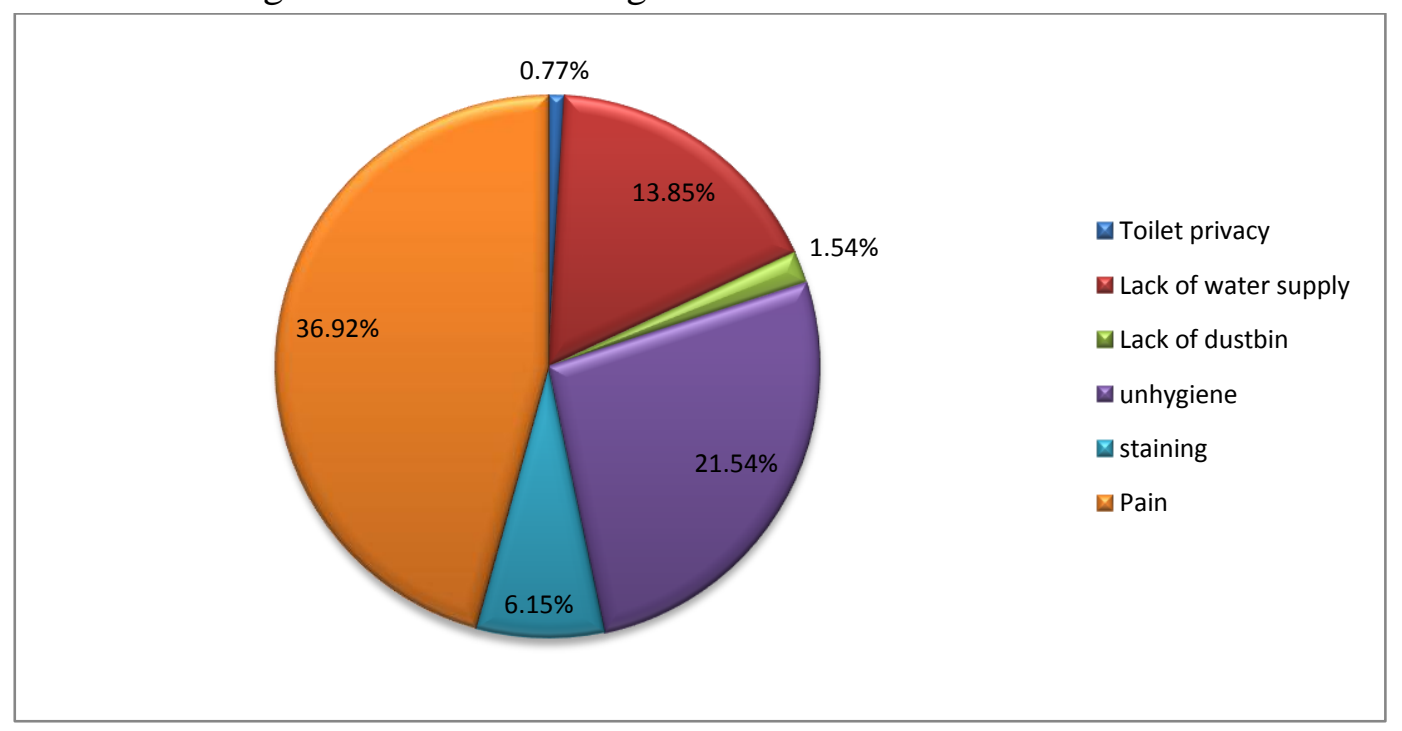

Figure 2: Activities Restricted During Menstruation among Study Participants

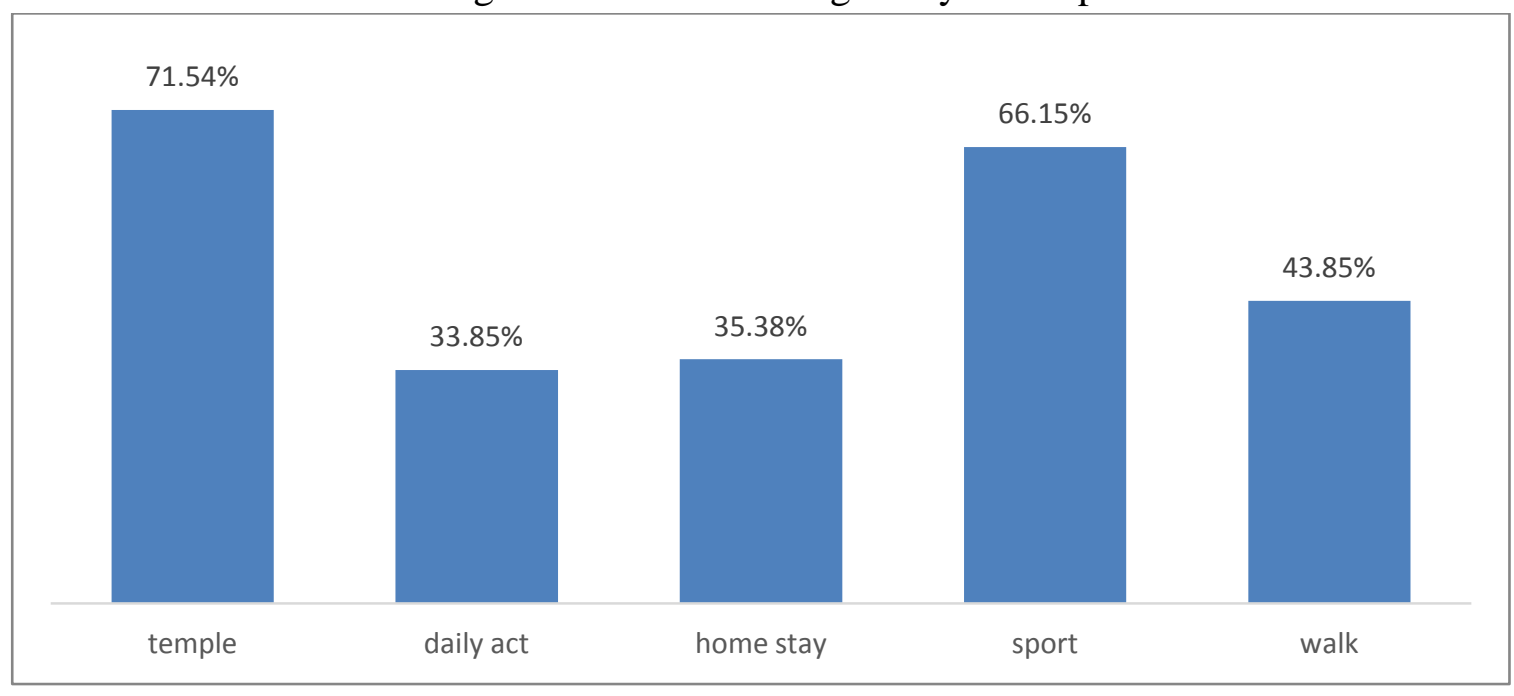


Figure 3: Distribution of premenstrual symptoms among study participants

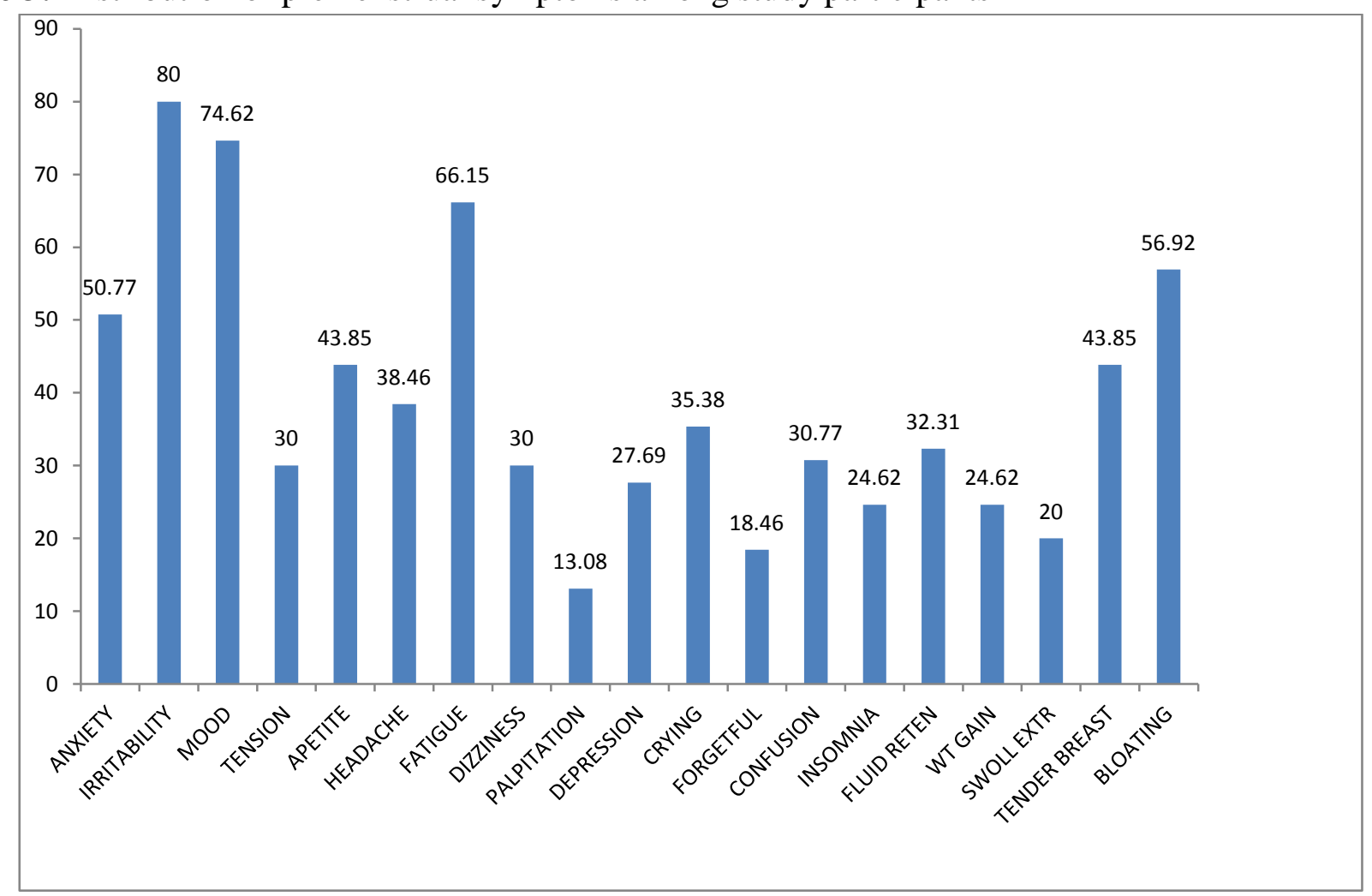

\section{Discussion}

This study was conducted among female medical students therefore majority of them had good knowledge on menstruation which is found to be $82.31 \%$. This is in contrast to study done by Mohapatra et $\mathrm{al}^{[8]}$ where students had good menstrual hygiene knowledge. $76.15 \%$ girls knew about menstruation before attainment of menarche and this result is in accordance with other studies done in population other than medical students. ${ }^{[1,9,10]}$. But in a study done in rural adolescent girls of Nepal, the knowledge and practice were $40.6 \%$ and $12.9 \%$ respectively, according to Adhikari $\mathrm{P}$ et al 2007. ${ }^{[11]}$ It is evident from a study done by Kalpana Katiyar et al 2013 that awareness is only $38.5 \%$ among adolescent females in an urban area of Meerut. ${ }^{[12]}$ The reason for unawareness among rural girls could be lack of educational background. In our study mean age of attaining menarche was 13 years which are almost similar to other studies done in different population population (urban, rural, and tribal) in various places all over the India. ${ }^{[1,10,13]}$. In this study $98.46 \%$ of the girls were using sanitary pads as menstrual absorbent material, other studies ${ }^{[14]}$ shows similar results. But in older studies, a higher use of cloth as absorbent was found. ${ }^{[1,13]}$. In our study the prevalence of college absenteeism was $45 \%$ which is similar to the study done by mohopatra et al where it was $49 \% .{ }^{[8]}$ In our study students followed a lot of restrictions like $71.54 \%$ girls restricted to go to temple, $66.15 \%$ were unable to do sport, $43.85 \%$ were unable to walk etc. Such practice of taboos were also found in other studies like Dasgupta et al, Thakre SB et al Sharma $\mathrm{N}$ et alas well, ${ }^{[1,13,15]}$ but in our study the number was found to be higher. Irritability is the most common premenstrual symptom in our study followed by mood swings. A large number of studies have been done on practices of menstrual hygiene, but there is a paucity of studies among health care professionals, hence we took this study.

\section{Conclusion}

Menstrual hygiene, is a vital aspect of health education. Good menstrual hygiene is crucial for the health, education, and dignity of girls and women. It is a social issue that cannot be addressed by working in schools and colleges 
alone. Education about the significance of menstruation and development of secondary sexual characteristics, selection of a sanitary menstrual absorbent and its proper disposal should be inculcated from early school days. This can also be achieved through educational television programmes, and knowledgeable parents. In order to ensure that adolescent girls and women have the necessary support and facilities, it is important that the wider society, communities and families must challenge the status quo and break the silence around menstruation.

\section{References}

1. A Dasgupta and M SarkarMenstrual Hygiene: How Hygienic is the Adolescent Girl? Indian J Community Med. 2008 April; 33(2): 77-80.

2. Lawan UM, Nafisa Wali Yusuf, Aisha Bala Musa. Menstruation and menstrual hygiene amongst adolescent school girls in Kano, northwestern Nigeria.African journal of reproductive health. 2010; 14(3):201-208

3. National Guidelines, Ministry of Drinking Water and Sanitation, Government of India,; Menstrual Hygiene Management; Paryavarn Bhawan, CGO Complex Lodhi Road, New Delhi-110003; December 2015; p. 1-36.

4. Oxford Living Dictionaries [Internet]. Available from: https://en.oxforddictionaries.com/definition /absenteeism

5. Dawn CS. Textbook of Gynaecology and Contraception. 10th ed. Calcutta: Dawn Books; 1990.

6. Waite LJ. US women at work. Population Bull. 1981;36:3.

7. Tegegne TK, Sisay MM. Menstrual Hygiene Management and School Absenteeism among Female Adolescent Students in Northeast Ethiopia.BMC Public Health 2014, 14:1118.

8. Mohapatra I, Roy A. A study of menstrual hygiene management and college absenteeism among female medical and dental students in a university of East India. Int J Health Sci Res. 2016; 6(12):21-27.

9. Deo DS, Ghattargi CH. Perceptions and practices regarding menstruation. A comparative study in urban and rural adolescent girls. Indian Journal of Community Medicine. 2005;30:33-34.

10. Juyal R, Kandpal SD, Semwal J, Negi KS. Practices of menstrual hygiene among adolescent girls in a District of Uttarakhand. Indian Journal of Community Health.2012 April-June 2012; 24(2): 124128.

11. Adhikari P, Kadel B, Dhungel SI. Knowledge and practice regarding menstrual hygiene in rural adolescent girls of Nepal. Kathmandu Univ Med J (KUMJ). 2007 Jul-Sep;5(3): 382-6.

12. Kalpana Katiyar, Harivansh Chopra, Sunil Kumar Garg. KAP study of menstrual problems in adolescent females in an urban area of Meerut. Indian $\mathbf{J}$ Community Health. 2013;25(3):217-220

13. Subhash B. Thakre, Sushma S. Thakre, Monika Reddy. Menstrual hygiene: knowledge and practice among adolescent school girls of Saoner, Nagpur district. Journal of Clinical and Diagnostic Research. 2011; 5:1027-103.

14. Juyal R, Kandpal SD, Semwal J, Negi KS. Practices of menstrual hygiene among adolescent girls in a District of Uttarakhand. Indian Journal of Community Health.2012 April-June 2012; 24(2): 124128

15. Sharma N, Sharma P, Sharma Net al.A cross sectional study of knowledge, attitude and practices of menstrual hygiene among medical students in north India. The Journal of Phytopharmacology 2013; 2(5): 28-37. 